Cinémas

Revue d'études cinématographiques

Journal of Film Studies

\title{
Du fantastique littéraire au fantastique filmique : une question de point de vue?
}

\section{Christiane Lahaie}

Volume 5, numéro 3, printemps 1995

Cinélekta 1

URI : https://id.erudit.org/iderudit/1001146ar

DOI : https://doi.org/10.7202/1001146ar

Aller au sommaire du numéro

Éditeur(s)

Cinémas

ISSN

1181-6945 (imprimé)

1705-6500 (numérique)

Découvrir la revue

Citer cet article

Lahaie, C. (1995). Du fantastique littéraire au fantastique filmique : une question de point de vue? Cinémas, 5(3), 45-63.

https://doi.org/10.7202/1001146ar
Résumé de l'article

Le texte littéraire fantastique déploie toute une batterie de stratégies narratives propres à en assurer l'intelligibilité. Au-delà d'un fantastique canonique, reconnu d'emblée comme tel, il existe un fantastique plus discursif, où l'acte d'énonciation et ultimement la narration tout entière se voit contaminés par une certaine ambiguïté. Or, ce type de fantastique scriptural issu d'un focalisateur plus ou moins « digne de confiance » existe-t-il toujours une fois adapté à un processus d'énonciation apparemment plus " objectif " comme celui du récit filmique ? L'analyse de deux textes littéraires fantastiques (Les Fils de la vierge de Julio Cortázar et The Haunting of Hill House de Shirley Jackson) et de leur adaptation pour le cinéma (Blow-Up, 1966, de Michelangelo Antonioni et The Haunting, 1963, de Robert Wise) nous fournira quelques éléments de réponses. 


\section{Du fantastique littéraire au fantastique filmique: une question de point de vue?}

\section{Christiane Lahaie}

\section{RÉSUMÉ}

Le texte littéraire fantastique déploie toute une batterie de stratégies narratives propres à en assurer l'intelligibilité. Au-delà d'un fantastique canonique, reconnu d'emblée comme tel, il existe un fantastique plus discursif, où l'acte d'énonciation et ultimement la narration tout entière se voit contaminés par une certaine ambiguïté. Or, ce type de fantastique scriptural issu d'un focalisateur plus ou moins "digne de confiance" existe-t-il toujours une fois adapté à un processus d'énonciation apparemment plus "objectif " comme celui du récit filmique? L’analyse de deux textes littéraires fantastiques (Les Fils de la vierge de Julio Cortázar et The Haunting of Hill House de Shirley Jackson) et de leur adaptation pour le cinéma (Blow-Up, 1966, de Michelangelo Antonioni et The Haunting, 1963, de Robert Wise) nous fournira quelques éléments de réponses.

\section{ABSTRACT}

In fantastic literature, the text deploys a whole battery of characteristic narrative strategies to ensure its intelligibility. Beyond the immediately recognizable fantastic canon, there exists a more discursive fantastic, in which the act of enunciation, and ultimately the entire narration, is tainted by a certain ambiguity. But can this type of fantastic writing, stemming from such an untrustworthy source, withstand adaptation to an apparently "objective" process of enunciation like the 
filmic narrative? An examination of two texts from fantastic literature (Julio Cortázar's Les Fils de la vierge and Shirley Jackson's The Haunting of Hill House) and their film adaptations (Michelangelo Antonioni's Blow $U_{p}, 1966$, and Robert Wise's The Haunting, 1963) will help us answer this question.

Depuis les tout débuts du cinéma, on s'est appliqué à adapter des œuvres littéraires fantastiques pour le grand écran: de Faust et Marguerite (1898) de Georges Méliès, en passant par Nosferatu le Vampire (1922) de Friedrich Wilhelm Murnau, jusqu'aux films plus récents de Brian DePalma et Francis Ford Coppola. Qu'il s'agisse d'un fantastique d'horreur ou d'un onirisme plus nuancé, il continue d'évoluer avec le temps, les pratiques d'écriture et les nouvelles possibilités offertes par la technologie cinématographique.

Or, bien que le fantastique littéraire bénéficie de nombreux apports critiques comme ceux de Tzvetan Todorov, Irène Bessière et Rosemary Jackson, les recherches sur le cinéma spécifiquement fantastique s'avèrent plutôt rares, surtout en ce qui a trait aux enjeux formels entraînés par l'avènement du fantastique moderne. Les études thématiques ne parvenant pas toujours à rendre compte de la complexité de ce nouveau fantastique, il faut désormais fureter du côté de sa narrativité, narrativité que les travaux de Gérard Genette, Mieke Bal, André Gaudreault et François Jost ont trop souvent négligée. De plus, s'il est vrai qu'on adapte une multitude de récits scripturaux fantastiques pour le cinéma, il vaudrait peut-être la peine de s'interroger sur le type de fantastique qu'on choisit majoritairement. On s'en doute: il s'agit d'un fantastique que l'on pourrait qualifier de "canonique", où défile tout le bestiaire fantastique habituel, soit les vampires, les goules, les fantômes, etc. '. Or, en littérature, le point de vue d'un personnage, qu'il soit narré ou narrateur, peut assurer à lui seul l'intégrité de l'événement fantastique ${ }^{2}$. Qu'un focalisateur omniscient vienne ou non sanctionner ce même point de vue ne compromet en rien la cohésion du texte puisque le point de vue subjectif du personnage continue généralement de primer. Le récit littéraire peut donc bénéficier de formes de 
modalisation propres à faire surgir le fantastique à même ses figures textuelles, indépendamment de la thématique fantastique habituelle.

On constate rapidement que les cas d'adaptation du fantastique moderne au cinéma s'avèrent moins fréquents et si d'aventure quelque réalisateur se risque à le faire (il y en a!), le produit final tend à basculer du côté du fantastique canonique (The Innocents, 1961, de Jack Clayton) ou du "drame" (Il deserto dei Tartari, 1976, de Valerio Zurlini). Serait-ce que le récit filmique n'a pas la possibilité de traduire le point de vue irréductiblement subjectif requis par le fantastique moderne? Ne confine-t-il pas la narration / focalisation à un rôle essentiellement objectif à cause de l'intervention inévitable d'un œil mécanique?

Afin de traiter du littéraire et du filmique comme des véhicules d'une focalisation objective ou subjective à même l'acte de narration, nous avons retenu deux types de récit littéraire fantastique ayant fait l'objet d'une adaptation filmique. Il s'agit premièrement d'un récit au "il" et au "je", à focalisation double (celle d'un focalisateur omniscient et celle d'un personnage à qui on délègue régulièrement la focalisation), un récit où, en principe, objectivité et subjectivité travaillent de concert, soit The Haunting of Hill House de Shirley Jackson. Scénarisé par Nelson Gidding et réalisé par Robert Wise, The Haunting (1963) est connu sous le titre français de La Maison du diable. Deuxièmement, nous étudierons un récit au "je", à focalisateur unique et entièrement conditionné par la subjectivité d'un personnagenarrateur dont la perception des choses peut s'avérer juste ou non, puisqu'il n'a accès qu'à sa seule "réalité ": la nouvelle Les Fils de la vierge de Julio Cortázar dont Michelangelo Antonioni et Tonino Guerra ont tiré un scénario qui a été porté à l'écran par Antonioni, sous le titre de Blow-Up (1966).

\section{Les cyclopes du fantastique littéraire}

Dans sa préface aux Récits fantastiques de Théophile Gautier, Marc Eigeldinger s'arrête longuement aux implications du regard et de la vision dans la littérature fantastique. Il mentionne, entre autres, la double nature du regard dont est doté le narrateur fantastique :

Du fantastique littéraire au fantastique filmique: une question de point de vue? 
Le regard ne possède pas seulement la faculté de la transparence, il est défini par son aptitude au dédoublement: "l'œil charnel" en tant qu'organe de la vue externe et "l'œil de l'âme", capable de percevoir la vision intérieure. Ce don de la voyance permet de décrypter le sens des choses du passé, de découvrir par divination la vérité du futur et d'enrichir le réel de la dimension de l'imaginaire (Gautier, p. 22).

Deux types de focalisation entrent en scène ici : d'une part, la perception sensorielle et, d'autre part, une vue tournée vers l'intérieur et plus subjective. Le fantastique littéraire exploiterait tacitement les ressources combinées de ces deux points de vue dans le but de rendre compte, non pas tant de la réalité objective que d'une "réalité" filtrée par une conscience potentiellement trouble. Aussi, depuis que de nombreux écrivains utilisent le point de vue d'un narrateur qui peut se tromper ou mentir, cet élément clé de la narrativité moderne fait l'objet de nombreux débats. Dans son article intitulé "Distance et point de vue", Wayne C. Booth pose les jalons de sa théorie en présentant deux grandes catégories de narrateur: le digne de confiance (reliable) et l'indigne de confiance (unreliable). Le fantastique, tel qu'on le conçoit souvent désormais, un fantastique intérieur, narcissique et qui fait surgir les monstres du dedans et non plus du dehors, obéirait à un impératif narratif crucial, celui du point de vue à objectivité (ou subjectivité) variable :

Les points de vue intérieurs sont donc soumis à des variations $[\ldots]$; ces variations sont encore plus importantes, selon le degré de confiance que l'on accorde au narrateur. D'une façon générale, plus l'incursion est profonde, plus nous acceptons d'être trompés, sans pour cela que notre sympathie faiblisse (Booth, p. 110).

Il semble, en effet, que le lecteur de fantastique tende à croire un narrateur sincère au point de se livrer en pâture aux foudres des bien-pensants, même un qui n'hésite pas à avouer ses pulsions de meurtre comme c'est le cas dans The Cask of Amontillado ou The Tell-Tale Heart d'Edgar Allan Poe.

On sera tenté de limiter la question du point de vue (et de son corollaire, l'identification au personnage) par le recours à la 
personne grammaticale. Le "il" correspondrait de facto à une narration objective et le "je", à une narration dont il faut à tout prix se méfier. S'inspirant des travaux de Booth dans Rhetoric of Fiction, Bruce Morrissette évite une conclusion aussi hâtive. Pas plus que le "il " ne garantit une omniscience réelle, le «je» de la narration moderne peut présenter des caractéristiques telles qu'il devient un "je" aliéné, très proche du "il». Ce "je» d'un autre aurait pour effet d'initier une médiation déterminante entre instance de narration et narrataires plus ou moins implicites. Il constitue :

[...] un moyen de créer l'aliénation, par la paralipse ${ }^{3}$, la non-fiabilité, un double registre pronominal et temporel [dualité entre temps de l'action et temps du récit] ainsi que d'autres méthodes visant à produire une tension entre le narrateur et le lecteur, entre l'identification et le rejet, entre la sympathie et l'aliénation (Morrissette, p. 101).

Lorsque ce "je" décide de faire cavalier seul, en focalisation interne ${ }^{4}$, il reste l'unique témoin du fantastique de sorte que son récit risque d'acquérir le statut de fruit probable d'une imagination débridée. Aussi, la confiance apparemment inconditionnelle qu'un lecteur de fantastique accorde au narrateur-focalisateur dépend plutôt de la nature intrinsèque de ses principaux focalisés: sentiments de crainte, de doute ou d'incrédulité, conflits intérieurs, bref, que de l'imperceptible. Or, il s'agit là précisément de la principale lacune du récit filmique. Comment illustrer le fantastique intérieur sans changer le cours des choses puisque, désormais, on doit transformer la moindre émotion en un acte bien perceptible, d'abord par la caméra, puis par le spectateur? Est-ce à dire que le filmique se révèle incapable de subjectivité? Faut-il conclure que l'objectif y règne en roi et maître au détriment de la subjectivité et que, par conséquent, certains types de fantastiques littéraires seraient impossibles à rendre au cinéma?

\section{L'œil sans paupière du fantastique filmique}

Qualifiant le cinéma d' «image trafiquée d'une réalité déjà trafiquée", Gaudreault met en lumière le double mensonge du 
récit filmique, un double mensonge qui s'apparente étrangement à la double vision prêtée au narrateur fantastique par Eigeldinger. De même que le narrateur littéraire peut mentir, le méganarrateur a la possibilité de "personnaliser" son champ. Selon Béla Balazs, non seulement la caméra le fait, mais elle parviendrait à rendre les images subjectives dans toute leur authenticité. La prise de vue combine bel et bien deux registres de "réalité " dans la simultanéité du plan:

Ces variations [de points de vue] sur une même image [...] sont [...] des représentations objectives des émotions subjectives des personnages. Après tout, on montre des images oniriques au cinéma. Les images oniriques sont si subjectives qu'elles ne peuvent représenter une réalité extérieure [au personnage qui les rêve]; elles sont nées de l'iconographie interne de la mémoire. Néanmoins, rêver est un phénomène naturel, existant dans la réalité objective, le matériau spécifique de l'art cinématographique (Balazs, p. 95).

Cependant, alors que le fantastique littéraire moderne autorise un usage de la focalisation en tant que filtre essentiel à l'apparition du surnaturel, le fantastique filmique n'a d'autre choix que d'utiliser l'ocularisation pour fournir au spectateur en plein ce qu'il réclame: un miroir dont l'activité déformante soit bien dissimulée. Aussi, on comprendra qu'à cause du dispositif de prise de vue obligé du cinéma, le problème de la focalisation dans le fantastique filmique se pose en ces termes: un personnage peut mentir ou avoir des lubies, ce que la caméra ne peut pas faire, du moins en principe 5 . Or, comme le précise justement Gaudreault, la caméra a plus de facilité à déguiser sa subjectivité, ce qui ne l'empêche pas de pouvoir l'exercer en temps voulu :

[...] les effets d'anthropomorphisme sont sûrement plus faciles à neutraliser dans le champ du cinéma, étant donné le caractère proprement "machinique " de son appareil de base... Même s'il faut un humain pour appuyer sur le bouton-déclencheur de la caméra [...] l'énoncé "photogrammique" (la suite de photogrammes que constitue un plan) n'apparaît jamais au départ 
comme un énoncé d'origine anthropomorphe, au contraire de l'énoncé linguistique [...] (1988b, p. 177).

Le langage filmique exerce donc deux activités distinctes et simultanées, concourant à assurer la circulation des points de vue: l'ocularisation "objective", opérée par la caméra même, et la focalisation "subjective", qui met l'accent sur le point de vue adopté par la narration filmique. Dans L'CEil-caméra, Jost précise que l'ocularisation devient interne et subjective lorsqu'elle passe par un personnage. Le spectateur aura droit, à l'occasion, à de l'ocularisation interne primaire, ou caméra subjective, laquelle suggère le regard d'un personnage précis en en dénotant l'état physique ou psychologique particulier (myopie, ivresse, démence). L'usage de la caméra subjective pour transmettre le point de vue unique d'un personnage-narrateur peut s'avérer une solution intéressante dans le cas du fantastique, mais elle doit demeurer provisoire puisque l'employer du début jusqu'à la fin d'un film équivaudrait à rester en ocularisation zéro, sans variation de points de vue.

Cela explique sans doute pourquoi les réalisateurs de films fantastiques se sont souvent rabattus sur le niveau d'identification dit secondaire et qui traduirait le "désir fondamental d'entrer dans un récit " (Aumont et Marie, p. 187). Jost parle d'ocularisation interne secondaire, "[...] lorsque la subjectivité d'une image est construite par le montage, les raccords (comme dans le champ/ contrechamp) ou par le verbal (cas d'une accroche dialoguée), en bref, par une contextualisation" (p. 23). Ce type d'ocularisation permet de montrer, en alternance, le sujet regardé et le sujet regardant et d'établir entre eux un rapport cognitif étroit. Ce choix de focalisation pourrait s'avérer une méthode valable dans la transmission du savoir fantastique. Tel que cité dans L'Analyse des films par Jacques Aumont et Michel Marie, Jean Narboni décrit les effets d'une telle focalisation, particulièrement prisée par Alfred Hitchcock dans la plupart de ses films :

[Les personnages] ne sont pas seulement mus par les affects ou par des sentiments, mais par une passion interprétative et une fièvre de déchiffrement qui peut 
aller, dans les plus grands films, jusqu'au délire de la construction d'un monde mettant à mal le principe de réalité (p. 28).

Ainsi, adhérer de façon univoque au point de vue d'un personnage rétrécit, en quelque sorte, le coefficient de réalité mis à la disposition du spectateur, sans neutraliser le réel pour autant. En revanche, la tendance "objectivisante" du cinéma tient du fait qu'il ocularise en premier lieu, pour ne focaliser qu'ensuite, même en situation de caméra subjective (un simulacre de subjectivité qui reste, lui aussi, d'abord et avant tout ocularisation). Si le fantastique littéraire permet une aliénation totale, le fantastique filmique impose des balises à cette même aliénation de par l'ocularisation initiale de la caméra. Certains récits littéraires, où le fantastique dépend de la subjectivité d'un personnage, doivent passer d'une focalisation unique à une focalisation double (ou multiple) et réputée fiable. Ainsi, le tournage ne pourra éliminer l'objectivité inhérente au filmique, même en privilégiant la focalisation d'un personnage, sans que l'ocularisation ne vienne tôt ou tard revendiquer ses droits. La focalisation mise en place par le film, même si elle est attribuée à l'un ou l'autre des personnages, passe par l'ocularisation zéro, une vision dont l'acuité ne saurait traduire l'incertitude. Or, un jour...

\section{The Haunting ou le réalisme fantastique}

Eleonor Vance, une jeune femme apparemment dotée de pouvoirs télékinésiques, se rend à Hill House où l'a convoquée le professeur Montague, afin de mener une enquête sur un phénomène paranormal: Hill House est hantée. Eleonor se prend d'affection pour la maison, qu'elle refuse de quitter, tant et si bien qu'elle meurt dans un accident de voiture, écrasée contre un arbre du jardin.

Dans le roman de Jackson, deux instances de focalisation conjuguent leurs efforts pour mener le récit à terme. Une première instance, le focalisateur omniscient, dont la présence ne se dément jamais du début à la fin, conclut le récit comme il l'avait entamé en focalisant sur la maison soi-disant hantée: 


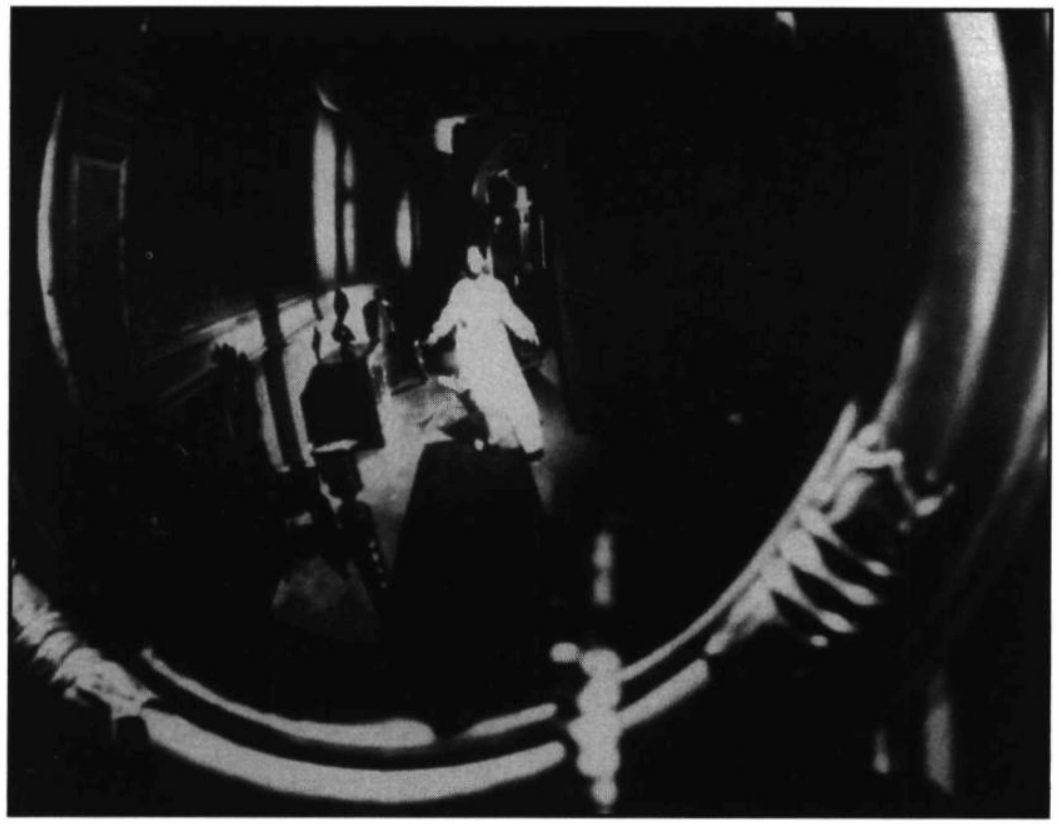

\section{The Haunting de Robert Wise (1963)}

\section{Collection Cinémathèque québécoise}

Hill House itself, not sane, stood against its hills, holding darkness within; it had stood so for eighty years and might stand for eighty more. Within, its walls continued upright, bricks met neatly, floors were firm, and doors were sensibly shut; silence lay steadily against the wood and stone of Hill House, and whatever walked there, walked alone.

Le narrateur affiche une omniscience relative puisqu'il ne révèle jamais la cause exacte des événements fantastiques de Hill House, la maison "malade». De plus, le fait qu'il délègue souvent la focalisation à Eleonor contribue à rehausser la saveur moderne de ce récit fantastique.

A en juger par le travail de "refocalisation " effectué par Wise dans l'adaptation du roman The Haunting of Hill House, il y a moyen de minimiser les dégâts et de maintenir, pour un temps du moins, l'ambiguité du fantastique moderne en concentrant davantage l'ocularisation / focalisation sur le personnage d'Eleonor

Du fantastique littéraire au fantastique filmique : une question de point de vue? 
Vance, personnage qu'on aura pris le soin de caractériser comme vaguement névrosé. Dans le récit filmique, la première instance de focalisation du roman se dédouble, scindée en deux focalisateurs qui se manifestent chacun à leur façon. D'abord, le mégamonstrateur ${ }^{6}$ cadre "objectivement" la maison en plan d'ensemble et contre-plongée, ensuite, une voix off (en l'occurrence celle de l'instigateur de toute cette partie de plaisir $\left.{ }^{7}\right)$ énonce "subjectivement" l'introduction citée plus tôt et dont le sens véritable ne pourra être décodé qu'en bout de course. Après avoir fait l'historique plutôt sombre de la maison, le professeur Markway acquiert un statut de focalisateur équivalent à celui des autres personnages (Eleonor exceptée).

La seconde instance de focalisation mise de l'avant par Jackson, et que le narrateur omniscient privilégie, revêt les traits et la conscience trouble d'Eleanor Vance elle-même. Passant du statut de focalisée principale à focalisatrice, en externe sur les autres, en interne sur ses propres réactions, elle entache l'intégrité de la narration de sa subjectivité toute-puissante, tant et si bien que le lecteur n'arrive pas à établir un rapport causal satisfaisant entre l'événement fantastique et l'entité responsable de celui-ci. Est-ce que l'événement fantastique a vraiment lieu ou est-ce Eleanor qui l'imagine, ou mieux, le provoque grâce à ses dons particuliers? Le doute atteint son paroxysme lorsque les autres personnages du roman éconduisent poliment Eleanor parce qu'elle nuit à l'objectivité de leur démarche "scientifique». Si on oublie la maison hantée pour un instant, n'est-ce pas le monologue intérieur d'Eleanor qui occupe tout l'espace textuel? Quand elle dit: "Oui, je viens", n'avoue-t-elle pas sa complicité avec la maison qui, selon elle, veut la retenir à tout prix ?

Dans la version filmée, Wise calque ce procédé typiquement littéraire qu'est la transmission directe des pensées d'un personnage. En ayant recours à la voix over, il nous autorise à entendre les pensées d'Eleonor; il s'assure que cette focalisatrice, tout en décrivant ses craintes et ses espérances, oblige le spectateur à rattacher les phénomènes étranges qui se manifestent à Hill House au désir prégnant d'Eleanor de s'y installer à demeure. On a donc affaire à une focalisation double et simultanée, en interne par l'intermédiaire de la voix over, en externe par l'ocularisation de la caméra. 
Il ne faudrait pas croire que la focalisation externe n'ait pas acquis, elle aussi, ses lettres de noblesse quand il s'agit de transmettre le fantastique. Elle le ferait toutefois autrement, pour faire passer un fantastique décentré, où le sort du personnage n'inquiète plus autant que la peur suscitée par le cadrage, ou mieux, l'absence de cadrage de l'entité fantastique. Gaudreault et Jost soutiennent que " [...] la focalisation externe n'est jamais plus forte que lorsqu'on donne à voir une rétention de savoir " (p. 140) et c'est exactement par le biais de ce mode de focalisation que la plupart des occurrences fantastiques nous parviennent dans The Haunting. Le fantastique, dans ses manifestations tangibles, est perçu de tous, mais par l'ouïe seulement. Une grande part de l'intérêt de ce film consiste d'ailleurs à ne jamais montrer ce que tout concourt pourtant à focaliser avec insistance. La peur, et non plus le doute, devient alors le moteur du fantastique, comme l'indique Michel Chion dans Écrire un scénario:

[...] le cinéma, art du hors-champ et du non-montré a
développé particulièrement le ressort de la crainte, sous
la forme de la peur, dans toutes ses nuances, du frisson
à l'horreur en passant par l'angoisse et l'épouvante. On
pourrait dire que nulle forme dramatique n'a été mieux
équipée que le cinéma pour créer la peur: depuis ses
conditions matérielles de représentation (une salle
complètement obscure) jusqu'à son langage même
(fondé sur le non-vu ou le non-encore-vu: ce qu'on ne
voit pas inspire beaucoup plus la peur que ce que l'on
voit) (p. 118).

Bien peu de cinéastes ont cependant résisté à la tentation de montrer le fantastique, une tentation à laquelle a pourtant résisté Wise, au risque de voir son œuvre qualifiée de "film radiophonique" par nul autre que Stephen King. A cet égard, l'inscription sur le mur du hall que le film présente en focalisation interne secondaire (parce qu'elle est d'abord ocularisée par Luke puis montrée à tous) constitue une trace visible du fantastique, mais de quel fantastique au juste? D'un fantastique canonique (celui du fantôme d'Abigail, la fillette terrorisée qui a grandi à Hill House) ou d'un fantastique moderne (la possible aliénation d'Eleonor, cette fillette déguisée en femme)? 
Afin de créer l'équivalent d'un fantastique résolument moderne pour le filmique, une démarche plus audacieuse consisterait peutêtre à nier l'événement fantastique et à "limiter " les points de vue à un glissement progressif de l'objectivité-ocularisation zéro vers une subjectivite-focalisation interne secondaire exclusive et centrée sur un événement aux apparences réalistes. C'est le défi qu'a relevé Antonioni en adaptant Les Fils de la vierge de Cortázar.

\section{Blow-Up ou la réalité fantastique}

Roberto Michel prend des photos dans un parc. Après avoir tiré des agrandissements de ses clichés, il se rend compte qu'il a empêché un crime de se commettre. Bientôt, Roberto raconte que l'homme de la photo se met à bouger et allonge les bras pour l'agresser.

La nouvelle de Cortázar se déroule totalement et explicitement dans la tête de Roberto, son protagoniste. Ceci ne garantit pas l'absence d'événement fantastique, mais bien la présence d'un point de vue dédoublé: une focalisation simultanée à la fois subjective et extrêmement lucide, et une deuxième, après coup, objective mais plus restreinte. Ce procédé littéraire n'a rien de fantastique en soi. Ce qui le rend fantastique, c'est le paradoxe issu d'une subjectivité lucide et d'une objectivité aveugle. Si l'appareil-photo ne court aucun risque, Michel, lui, meurt d'avoir prêté vie à une image: "Mais les mains, c'en était trop. Je venais d'écrire: "Donc, la seconde clé réside dans la nature des difficultés que les sociétés ont à..." lorsque je vis la main de la femme se refermer lentement doigt après doigt " (Cortázar, p. 143). En tant que narrateur, Michel s'autorise à changer à volonté de personne grammaticale, sa focalisation se modifiant à l'avenant, toujours objective parce que témoignage d'une "prise de vue" (mais subjective aussi, ne serait-ce que par le choix de cadrage qui le fait inclure ou ne pas inclure tel ou tel élément de la "scène" dont il témoigne). Par son choix artistique et à travers le processus photographique, l'homme transforme une image en "tranche de fiction" dérobée à la réalité, une figure narrative que Stuart Y. McDougal met en relief au sujet du protagoniste des Fils de la vierge en soutenant que: 


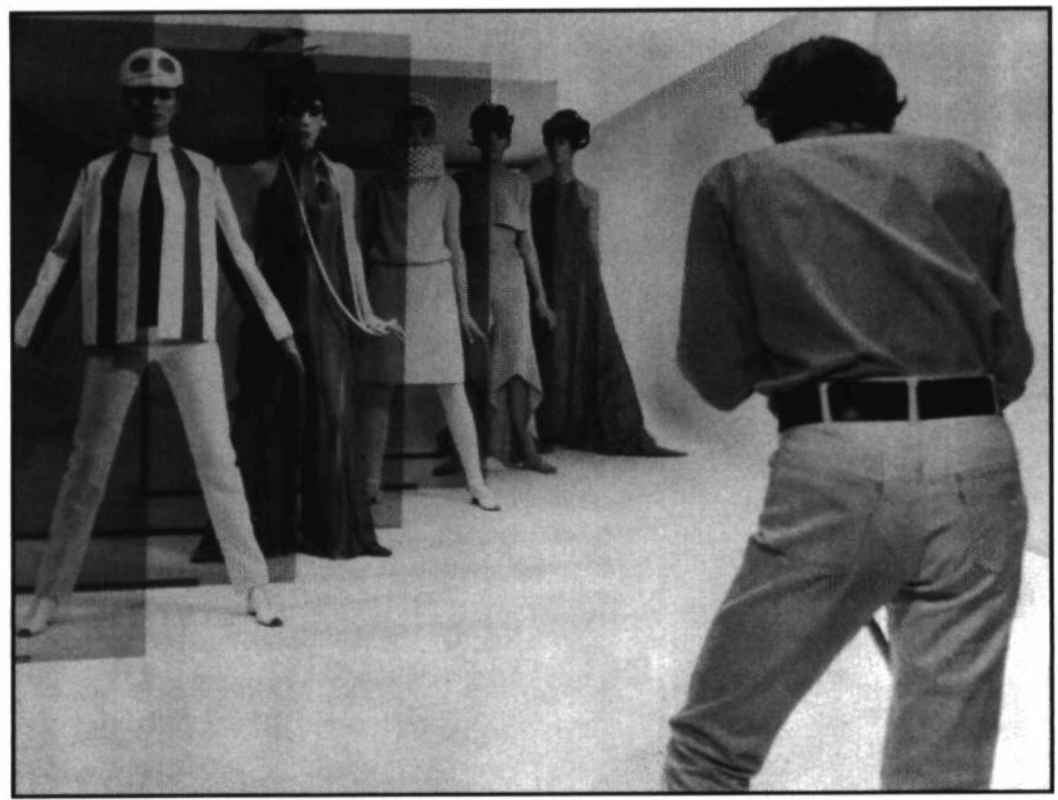

\section{Blow-Up de Michelangelo Antonioni (1 966)}

Collection Cinémathèque québécoise

[...] ses principes artistiques l'amènent à une distorsion de la situation. De plus, sa présence et le fait qu'il soit muni d'un appareil-photo perturbe la scène qu'il tente de photographier objectivement. De retour à son appartement et assis face à un agrandissement de la photo fixé au mur devant lui, Roberto doit confronter ces faits afin de bien comprendre ce dont il a été témoin (p. 224).

La main de Roberto guide l'œil froid de l'appareil-photo, lui accordant un semblant de subjectivité, exactement ce que fait Antonioni de sa caméra dans la mise en film de Blow-Up. Drame psychologique, étude de mœurs, intrigue policière, Blow- $U_{p}$ a porté tous les chapeaux sauf celui du fantastique. Pourtant, il constitue la mise en film de la conscience fantastique même, à savoir que le réel nous échappe nécessairement, surtout à partir du moment où on veut le cerner, l'encadrer, le délimiter.

Tout comme dans la nouvelle de Cortázar, le contenu diégétique de $B l o w-U_{p}$ paraît simple a priori. Par hasard, un photographe

Du fantastique littéraire au fantastique filmique: une question de point de vue? 
professionnel prénommé Thomas fixe sur pellicule un meurtre commis dans un parc public. Sur le coup, il ne remarque rien, sinon l'attitude bizarre de la femme photographiée contre son gré et qui veut absolument récupérer la pellicule. Le photographe, qu'une ocularisation zéro a déjà établi comme faisant preuve d'un esprit de contradiction hors du commun, s'obstine à conserver les photos qu'il a prises d'elle et de son amant. Plus tard, en les agrandissant et essentiellement par focalisation interposée (celle de la femme et comme s'il avait besoin de ses yeux à elle pour voir), il découvre qu'un autre homme se cachait dans les buissons, armé d'un revolver. La silhouette de cet homme reste cependant floue à cause du grain de la photo. À partir de ce moment, à l'aide d'agrandissements successifs, le photographe va tenter de comprendre ce qu'il perçoit réellement sur ses clichés, jusqu'à ce qu'il constate la présence d'un corps allongé sur le sol... Pendant que la nouvelle parle d'une impossibilité de dire et de savoir à travers les mots, le film exprime cette difficulté de voir et de comprendre à travers l'image.

Dans le film d'Antonioni, un récit à forme fantastique (à défaut d'en présenter la thématique habituelle), ce qui prend une importance démesurée, c'est le hors-champ que seule la focalisation interne secondaire sera en mesure de déchiffrer. Tout ce que l'ocularisation zéro avait empêché de voir sera confié aux bons soins de la focalisation interne et du champ personnalisé. En effet, l'ocularisation de l'instance de mise en cadre n'avait autorisé que des plans trop éloignés (dans le parc, puis sur l'agrandissement) pour que le spectateur ne puisse faire de découverte autrement qu'avec l'assistance d'une focalisation précise: celle de l'artiste contemplant son œuvre. Là où l'objectivité a échoué, la subjectivité donne des résultats. La focalisation déléguée au personnage de Thomas ne le quittera pas pendant toute la séance d'agrandissements-découvertes (allant même jusqu'à la caméra subjective à quelques reprises). Par des zooms avant et des panoramiques gauche-droite tentant d'imiter l'œil humain en train de comparer deux images, on s'assure qu'une subjectivité totale se greffe à l'ocularisation de la caméra et désire cerner la véritable teneur des photos. Ce procédé fonctionne au point que le spectateur fait la découverte du cadavre avec Thomas ${ }^{8}$. 
Antonioni reconnaît tacitement ici que le cinéma crée le sens à partir d'une dualité focale : une ocularisation d'abord (quitte à ce qu'elle soit plus ou moins dirigée) et une focalisation interne secondaire ensuite (qui, elle, s'empresse de diriger). Il crée le sens aussi à partir, et uniquement à partir, du contexte. Une photo seule ne signifie rien; toutes les photos regroupées rattrapent les possibles du hors-champ et signifient tout. Ainsi, la seule photo que les voleurs n'ont pas dérobée à Thomas, celle du cadavre isolé, allongé dans l'herbe, devient à peine décryptable à partir du moment où le contexte auquel on se devait de la relier disparaît.

Le fantastique de Blow- $U_{p}$, que d'aucuns se refuseront à admettre, se situe donc du côté de l'énonciation et non de l'énoncé. Ce récit filmique témoigne de la conscience fantastique et non du fantastique lui-même. Comme le précise Neil Isaacs dans son article "The Triumph of Artifice" auquel nous devons beaucoup, Thomas ne perçoit la réalité qu'à travers l'ocularisation de son appareil-photo: ses propres yeux ne peuvent que l'induire en erreur. N'est-ce pas pour palper le réel qu'il retourne au parc le lendemain du jour J, muni de son capteur d'images, et qu'il découvre que le cadavre a disparu? N'a-t-il pas compris la nature profonde du cheminement dans lequel il s'est engagé lorsqu'il appelle son ami pour lui dire qu'il a fait une découverte "fantastique" ?

À même un réseau sinueux de focalisations étudiées et jamais innocentes, Antonioni tisse une trame dont l'enjeu consiste à transposer le fantastique moderne, et entièrement subjectif, à l'écran. Nous croyons qu'il y parvient dans la mesure où il met en place les mécanismes de la conscience fantastique, celle qui accorde au réel une valeur fictive et qui fait de l'imaginaire un mode de fonctionnement plus proche de la vérité.

\section{Vers un fantastique du filmique?}

Ceux et celles qui entendent adapter le fantastique moderne pour le cinéma se retrouvent face à une double contrainte: celle de devoir montrer l'intériorité du personnage central supposément confronté au fantastique et celle d'avoir à composer avec l'objectif de la caméra. Par-delà une histoire des pratiques filmiques ${ }^{9}$, plus loin que la tradition cinématographique, le médium 
filmique impose la monstration ${ }^{10}$ et tous les fantastiques ne peuvent circuler au grand jour. Beaucoup de films fantastiques, bien qu'ils insufflent une certaine ambiguïté au point de vue de la narration, ne maintiennent pas cette ambiguïté jusqu'au bout parce qu'ils doivent montrer et non pas seulement suggérer. Si Wise refuse de révéler la véritable nature de Hill House, il fournit une explication précise à la mort d'Eleonor ". De même, parce que Blow- $U_{p}$ reste "discret" sur sa teneur fantastique, cette dernière se voit plus souvent qu'autrement ignorée.

Pourtant, le pouvoir suggestif de la caméra, pouvoir que le scénariste a le loisir de lui octroyer, existe bel et bien selon Jackson parce que

\begin{abstract}
[...] dans la traduction du genre fantastique pour le cinéma, ces problèmes [de points de vue de la narration] sont refocalisés autour de la vision de l'œilcaméra qui peut produire des représentations similaires d'enregistrements "objectifs" (ou documentaires) tout autant que des représentations de la présence d'une vision "subjective" par le biais d'un personnage du récit. Ou encore, il peut y avoir une représentation de combinaisons "irréelles " d'objets et d'événements, comme "réalistes", à travers l'œil même de la caméra - en ce sens - le processus cinématographique en soi pourrait être qualifié de " fantastique " (p. 31).
\end{abstract}

Par cette observation, Jackson mise sur la possibilité d'un fantastique spécifiquement cinématographique à partir de la dichotomie objectivité/subjectivité. Ce ne serait plus une technique de focalisation calquée sur celle du récit scriptural fantastique, mais une logique des regards qui tienne compte, à la fois, de l'objectivité inéluctable d'une caméra et de la subjectivité irréductible de l'œil qui se cache derrière elle.

Université Laval

\title{
NOTES
}

1 Dans les chapitres 2 et 3 de l'ouvrage de Tzvetan Todorov, Introduction à la littérature fantastique (Paris: Seuil, 1970), on trouvera une classification du fantastique en quatre catégories allant de l'étrange pur au merveilleux pur, en passant par le fantasti- 
que étrange et le fantastique merveilleux. Afin de dresser cette typologie, Todorov a arrêté de nombreux critères dont les principaux sont l'hésitation du lecteur (et éventuellement du personnage) devant une explication naturelle et une explication surnaturelle d'un événement fantastique. Alors que le merveilleux pur assure l'adhésion instantanée du lecteur (et des personnages) au surnaturel (par ex. La Belle au bois dormant), le fantastique commande une hésitation (par ex. Dracula). Quant à la définition de l'étrange pur, telle que fournie par Todorov, elle semble plus floue: « [...] on relate des événements qui peuvent parfaitement s'expliquer par les lois de la raison, mais qui sont, d'une manière ou d'une autre, incroyables, extraordinaires, choquants, singuliers, inquiétants, insolites et qui, pour cette raison, provoquent chez le personnage et le lecteur une réaction semblable à celle que les textes fantastiques nous ont rendue familière" (p. 51-52). Nous préférons délimiter le fantastique à la fois par le biais de ces thèmes et à travers les effets qu'il génère. Ainsi, le fantastique canonique, beau paradoxe s'il en est un, nous sera d'abord et avant tout familier: il vise à faire peur, à déstabiliser le lecteur-spectateur et utilise, ce faisant, des ressorts éprouvés (êtres repoussants aux dons surnaturels, lieux inquiétants, etc.). Il fait appel au bon vouloir du lecteur qui accepte de croire au fantastique et en redemande (nous pensons ici aux lecteurs de Stephen King). Quant à ce que nous appelons le "fantastique moderne" ", il s'agit d'un fantastique où notre quotidien même est remis en question, d'où l'angoisse. De plus, ce fantastique pourrait très bien n'exister que dans la tête d'un personnage auquel on est forcé de s'identifier, mais l'histoire ne le dit pas. Si le fantastique canonique, tel que nous le définissons est avant tout affaire de thèmes et se conclut sur des certitudes, le fantastique moderne est surtout affaire de point de vue et laisse le lecteur dans le doute. $\AA$ lui de décider si le fantastique est partout ou nulle part.

2 Le Tour d'ecrou d'Henry James constitue l'exemple le plus patent de ce type de fantastique. Jean-Baptiste Baronian dit de James "[...] qu'il situe l'inadmissible non plus nécessairement - non plus par habitude - à l'extérieur de l'homme, dans l'implacable décor que forment les choses du monde sensible, dans le champ usuel du regard, mais plutôt en l'homme lui-même" (Un nouveau fantastique, Lausanne: L'Âge d'Homme, 1977, p. 21). En 1961, cette nouvelle a d'ailleurs fait l'objet d'une adaptation cinématographique relativement réussie de la part des scénaristes William Archibald et Truman Capote de même que pour le réalisateur Jack Clayton. Dans Filming Literature: The Art of Screen Adaptation, Neil Sinyard prétend qu'en adaptant Le Tour d'ecrou de James, les scénaristes de Clayton ont pris le parti de raconter l'histoire d'une gouvernante aliénée. Mais afin de résoudre le problème de la transposition d'une focalisation subjective qui crée le fantastique pour un médium plus objectif, ils auraient décidé de faire basculer le rapport de cause à effet. Dans The Innocents, film dont le titre est déjà en soi une prise de position, on s'assure que le spectateur voie la gouvernante réagir physiquement à quelque chose avant que le quelque chose (Jessel sur le bord de l'eau, Quint sur la tour) ne soit montré à l'écran, de sorte que l'on croie que c'est bel et bien la gouvernante qui a des hallucinations. Jeanne Thomas Allen, dans un article intitule "The Turn of the Screw and The Innocents: Two Types of Ambiguity ", soutient que cette adaptation et son ambiguïté due au point de vue de Miss Giddens seraient mieux desservies par la bande sonore que par les images. Giddens paraît plus sensible aux bruits naturels (le bruissement des feuilles, le vent), on est donc poussé à croire, par ricochet, qu'elle a une "sensibilité extrême" la portant à attribuer le statut de réalité à tout ce qu'elle imagine. Toujours selon Allen, l'ambiguïté ne sera pas maintenue jusqu'au bout, en partie à cause du jeu trop exalté de Kerr.

3 Selon Genette, la paralipse se définit comme étant une "altération " qui consiste " [...] à donner moins d'information qu'il n'est en principe nécessaire " (1972, p. 211).

Du fantastique littéraire au fantastique filmique: une question de point de vue? 
4 On éliminera cependant d'emblée la focalisation externe du récit littéraire "béhavioriste", un type que l'on retrouve rarement, sinon dans certaines œuvres issues de l'ère du Nouveau Roman et qui risquerait, en fantastique, de créer un effet de distanciation si prononcé que le tout basculerait du côté de la parodie. Imaginons, à titre d'exemple, un récit où un personnage invariablement focalisé en externe fait l'acquisition d'un château hanté. On le voit sursauter chaque fois qu'il ouvre une porte parce qu'un fantôme se trouve derrière et cela constitue sa seule réaction apparente au fantastique. La focalisation externe ne permet que l'observation des attitudes extérieures d'un personnage (sans jamais révéler son tourment intérieur) et dessert évidemment la cause fantastique.

5 Dans The Novel and the Cinema (London: Tanting Press, 1975), Wagner précise toutefois que quelques rares réalisateurs ont fait mentir la caméra. Il cite The Other (1972) de Robert Mulligan comme un cas de cette manipulation visuelle peu orthodoxe. On se souviendra que ce récit filmique présente objectivement deux jumeaux à l'esprit apparemment tordu et qui, ensemble, font les quatre cents coups. Après un temps (vers le milieu du film), on apprend qu'un des deux frères est mort depuis longtemps. Cela revient à dire que la caméra a montré objectivement ce qu'un des deux jumeaux voyait subjectivement, à travers la lunette de son dédoublement de personnalité. Selon Wagner, le roman de Tom Tryon dont le film a été tiré avertissait le lecteur de ce fait beaucoup plus tôt dans le déroulement du récit.

6 Linstance de mise sur film telle que définie par Gaudreault dans Du littéraire au filmique. Système du récit (Paris: Méridiens/Klincksieck, 1988a).

7 Le docteur Montague du roman, le professeur Markway du film.

8 Le cadavre se trouvait bel et bien sur la photo, avant l'épisode sexuel auquel s'adonne le protagoniste avec deux filles, épisode qui permet par ailleurs au photographe d'acquérir du recul. Grâce à la technologie magnétoscopique, nous avons " triché" et vérifié, a posteriori et par arrêt sur image, la présence du cadavre sur la première occurrence du cliché à l'écran. Il s'y trouvait. Nous avons d'abord cru qu'Antonioni avait "joué " avec le spectateur au point de faire apparaître le cadavre sur la photo et inclure ainsi un événement fantastique, ce qu'il n'a pas fait.

9 Nous croyons que les pratiques filmiques consacrées en majeure partie par le cinéma hollywoodien limitent la portée du fantastique moderne à l'écran. Montrer a plus d'effet que suggérer de même que respecter des schèmes narratifs simples où tout est résolu en bout de course s'avère plus rentable que de dérouter le spectateur moyen par le biais d'un montage novateur.

10 Une notion que Gaudreault décrit comme précédant la narration (assurée, entre autres, par le montage) et qui conviendrait davantage au récit filmique.

11 Au volant de son automobile, Eleonor effectue un virage qui lui sera fatal en voulant éviter l'épouse du Dr Markway. Il serait intéressant, par ailleurs, d'étudier les implications psychosociologiques de ce dénouement puisque Eleonor était tombée amoureuse du docteur et que le film semble assez explicite sur ce point.

\section{B IB LIOGRAPHIE}

Aumont, Jacques et Marie, Michel. L'Analyse des films. Paris: Nathan, 1988.

Bal, Mieke. Narratologie. Paris: Klincksieck, 1977.

Balazs, Béla. Theory of the Film: Character and Growth of the New Art. New York: Dover, 1970.

Baronian, Jean-Baptiste. Un nouveau fantastique. Lausanne: L'Âge d'Homme, 1977. Bessière, Irène. Le Récit fantastique. La poétique de l'incertain. Paris: Larousse, 1974. 
Booth, Wayne C. «Distance et point de vue: essai de classification ». Poetrique du récit (collectif). Paris: Seuil (1977), p. 85-113.

Chion, Michel. Ecrire un scénario. Paris: Cahiers du Cinéma / I.N.A., 1985.

Cortázar, Julio. Les Fils de la vierge, Les Armes secrètes. Paris: Gallimard (1963), p. 125-148.

Gaudreault, André. Du littéraire au filmique. Système du récit. Paris: Méridiens / Klincksieck, 1988a.

Gaudreault, André. "Narrator et narrateur". Dans Michel Larouche (direction), Le Cint́ma aujourd'hui : films, théories, nouvelles approches. Montréal: Guernica (1988b), p. 173-184.

Gaudreault, André et Jost, François. Cinéma et récit II. Le Récit cinématographique. Paris: Nathan, 1990.

Gautier, Théophile. Récits fantastiques. Paris: Garnier-Flammarion, 1981.

Genette, Gérard. Figures III. Paris: Seuil, 1972.

Genette, Gérard. Nouveau Discours du récit. Paris : Seuil, 1983.

Isaacs, Neil. "The Triumph of Artifice: Antonioni's Blow-Up (1966) from the Short Story by Julio Cortázar ". Dans Andrew S. Horton et Joan Magretta (direction), Modern European Filmmakers and the Art of Adaptation. New York: Frederick Ungar (1981), p. 130-144.

Jackson, Rosemary. Fantasy: The Literature of Subversion. London: Methuen, 1981. Jackson, Shirley. The Haunting of Hill House. New York: Penguin, 1959.

James, Henry. The Turn of the Screw / Le Tour d'ecrou. Paris: Presses Pocket, 1989.

Jost, François. L'Eil-caméra. Entre film et roman. Lyon: P.U.L., 1987.

McDougal, Stuart Y. Made into Movies: From Literature to Film. New York: Holt Rinehart and Winston, 1985.

Morrissette, Bruce. Novel and Film: Essays in Two Genres. Chicago : University of Chicago Press, 1985.

Sinyard, Neil D. Filming Literature: The Art of Screen Adaptation. New York: StMartin's Press, 1986.

Thomas Allen, Jeanne. "Turn of the Screw and The Innocents: Two Types of Ambiguity n. Dans Gerald Peary et Roger Shatzkin (direction), The Classic American Novel and the Movies: Exploring the Link between Literature and Film. New York: Frederick Ungar (1977), p. 132-142.

Todorov, Tzvetan. Introduction à la littérature fantastique. Paris: Seuil, 1970.

Wagner, Geoffrey. The Novel and the Cinema. London: Tanting Press, 1975.

Du fantastique littéraire au fantastique filmique: une question de point de vue? 